\title{
AVALIAÇÃO DE FERRAMENTAS BIM PARA ELABORAÇÃO DE PROJETOS EXECUTIVOS DE ARQUITETURA ${ }^{1}$
}

\section{BIM TOOLS EVALUATION FOR EXECUTIVE ARCHITECTURAL DESIGNS}

\author{
Ari Monteiro \\ Dharma Sistemas, SP \\ ari mont@yahoo.com.br \\ Karina Matias \\ Escola Politécnica da USP, SP \\ karinamatias@usp.br
}

\begin{abstract}
Resumo
Há alguns anos, o desenvolvimento de projetos executivos de Arquitetura utilizando ferramentas BIM é uma realidade no mercado brasileiro. Embora existam diversas ferramentas BIM que propõem atender as demandas desta área algumas se destacam mais no Brasil, tais como, o Graphisoft ArchiCAD e o Autodesk Revit. Estas ferramentas representam gerações distintas de tecnologia e possuem fiéis seguidores de ambos os lados. Enquanto uma oferece melhor flexibilidade para elaboração de modelos BIM, a outra oferece mais recursos de modelagem paramétrica sem requerer de seus usuários conhecimentos em desenvolvimento de software. Muito se discute sobre qual destas ferramentas é a mais adequada para atender as necessidades dos projetistas de Arquitetura. O discurso dos desenvolvedores de ferramentas BIM muitas vezes está focado no marketing dos seus produtos e omite os detalhes práticos da utilização da tecnologia oferecida por eles no mundo real. $O$ objetivo deste trabalho é responder a pergunta: Qual dessas ferramentas oferece maior conformidade aos processos BIM envolvidos no desenvolvimento de projetos executivos de Arquitetura? $\mathrm{O}$ trabalho pretende contribuir com informações úteis às empresas de projeto que intencionam usar, ou que já utilizam a tecnologia BIM na elaboração de projetos executivos de Arquitetura.
\end{abstract}

Palavras-chave: Ferramentas BIM. Arquitetura. Projeto Executivo.

\begin{abstract}
Few years ago, development of architectural design using BIM tools is a reality in the Brazilian market. While there are several BIM tools that propose to meet the demands of this area, only a few stands out more in Brazil, such as, Graphisoft ArchiCAD and Autodesk Revit. These tools represent different generations of technology and have faithful followers of both sides. Although one of these tools offers better flexibility for BIM models development, the other offers more parametrical modeling resources, without requiring knowledge of its users in software development. There is debate about which of these applications is best suited to meet the needs of architecture designers. The discourse of the BIM tools developers is often focused on their products marketing and omits the practical detail of the technology usability offered by them in the real world. The objective of this study is to answer the question: Which of these tools offers greater compliance with BIM processes involved in the development of executive architecture projects? The paper aims to contribute useful information to design firms that intend to use or already use BIM to elaborate of executive architecture projects.
\end{abstract}

Keywords: BIM Tools. Architecture Design. Construction Documents.

1 MONTEIRO, A.; MATIAS, K. Avaliação de ferramentas BIM para elaboração de projetos executivos de Arquitetura. In: ENCONTRO BRASILEIRO DE TECNOLOGIA DE INFORMAÇÃO E COMUNICAÇÃO NA CONSTRUÇÃO, 7., 2015, Recife. Anais... Porto Alegre: ANTAC, 2015. 


\section{和}

\section{INTRODUÇÃO}

O desenvolvimento de projetos executivos de Arquitetura utilizando ferramentas BIM é uma realidade no mercado há alguns anos. Embora existam diversas ferramentas BIM que propõem atender as demandas desta área, algumas delas se destacam mais, tais como, o Graphisoft ArchiCAD e o Autodesk Revit.

Muito se discute sobre qual das ferramentas BIM existentes no mercado é a mais adequada para atender as necessidades dos projetistas de Arquitetura. $O$ discurso dos desenvolvedores destas ferramentas muitas vezes está focado no marketing dos seus produtos e omite os detalhes práticos da utilização dessas ferramentas no mundo real.

Problemas reais, tais como, a necessidade de desenvolver padrões de projeto (templates) e bibliotecas de componentes, ou a melhor estratégia de modelagem para lidar com problemas de desempenho na manipulação de modelos BIM complexos, só são abordados quando as empresas já adquiriram as ferramentas e se vêem obrigadas a gerenciar os problemas resultantes de suas escolhas.

O objetivo principal do presente estudo é responder à seguinte questão de pesquisa: Qual das ferramentas BIM avaliadas neste estudo oferece maior conformidade aos processos BIM envolvidos no desenvolvimento de projetos executivos de Arquitetura? Outro objetivo desta pesquisa é orientar sobre quais são os aspectos de implementação que as ferramentas BIM demandam de uma empresa de projeto de Arquitetura.

Para responder a essa questão foi realizado um estudo que consistiu em avaliar as duas ferramentas BIM mais utilizadas no Brasil, atualmente, o Graphsoft ArchiCAD e o Autodesk Revit, sob o ponto de vista de quem as utiliza realmente, ou seja, seus usuários em empresas de projeto de Arquitetura.

Este trabalho representa o resultado parcial da pesquisa de Mestrado de um dos autores, sobre a implementação da tecnologia BIM em empresas de projeto de Arquitetura.

\section{REVISÃO DA LITERATURA}

\subsection{IMPLEMENTAÇÃO BIM EM EMPRESAS DE ARQUITETURA}

Sob as novas demandas do mercado e o incentivo dos agentes da cadeia produtiva, as empresas de projetos passaram a ser estimuladas a corresponder às novas necessidades de seus contratantes e a planejar a implementação da tecnologia BIM. Diante deste cenário, as empresas de projeto de Arquitetura devem priorizar a inovação como estratégia de negócio e avaliar o retorno de seu investimento a curto e longo prazo.

Também devem ser consideradas as particularidades no processo de se projetar Arquitetura dentro do contexto do BIM. As mudanças na produção do trabalho trazem benefícios e dificuldades em todas as etapas de projeto desde a concepção ao detalhamento final do produto. Há a necessidade de análise e revisão dos processos tradicionais quando se decide usar a tecnologia BIM.

No Brasil observa-se desde 2007, de acordo com Souza et al (2013), um início de movimentação no setor privado para a implementação do BIM. Segundo Nakamura (2013), as grandes construtoras e incorporadoras brasileiras iniciaram uma série de projetos-piloto para avaliar a aplicabilidade desta tecnologia, visando o aumento de produtividade, redução de perdas, diminuição de prazos, maior assertividade nos orçamentos e melhoria na qualidade do produto imobiliário. 
Em Reis (2011) são apresentados resultados de implementações BIM realizadas entre 2004 e 2007 em quatro empresas de projeto de Arquitetura de diferentes portes. Foram relatadas as primeiras experiências, dificuldades, ganhos e algumas recomendações. Esses relatos demonstram que estas empresas foram pioneiras na implementação do BIM, se antecipando as necessidades do setor e colaborando na difusão e adoção dessa tecnologia. Elas passaram por uma revisão dos seus métodos de trabalho, reorganizaram suas equipes estabelecendo novas lideranças e responsabilidades e encontraram maneiras de continuar a produção interna de projeto enquanto, simultaneamente, implementavam a nova tecnologia em projetos-piloto.

Implementar BIM efetivamente requer significativas mudanças na maneira de trabalhar em quase todos os níveis dentro do processo do empreendimento. Não é suficiente apenas o aprendizado de novas ferramentas, mas também, deve haver a reinvenção do fluxo de trabalho, treinamento da equipe e atribuição de novas responsabilidades (ARAYICY et al, 2011). Além disso, a utilização da tecnologia BIM na prática é uma decisão de negócio, pois a rotina de trabalho de uma empresa vai requerer mudanças de processos (JERNIGAN, 2008).

Portanto, a adoção da tecnologia BIM exige um esforço grande na mudança de processos internos no negócio de uma empresa de projeto, que influenciam inclusive, na forma de vender e entregar seus serviços.

\subsection{PROCESSOS BIM EM EMPRESAS DE ARQUITETURA}

O Manual de Escopo de Projetos e Serviços da Arquitetura e Urbanismo (ASBEA, 2012), caracteriza o processo de projeto de Arquitetura como uma sequência de atividades distribuídas por seis fases, que estão relacionadas àquelas estabelecidas pela NBR 15.531: (a) Fase A - Concepção do Produto: Levantamento de dados, Programa de Necessidades e Estudo de Viabilidade; (b) Fase B - Definição do Produto: Estudo Preliminar, Anteprojeto, Projeto Legal; (c) Fase C - Identificação das interfaces: Projeto Básico; (d) Fase D Detalhamento dos projetos: Projeto Executivo; (e) Fase E - Pós-entrega do projeto; (f) Fase F - Pós-entrega da obra.

Os projetos de Arquitetura têm características particulares em seu desenvolvimento, com uma grande ênfase nas suas etapas iniciais, que envolvem viabilidade e concepção. $O$ uso de ferramentas CAD 3D (Sketchup, por exemplo) é uma prática que está se tornando comum entre alguns Arquitetos nestas etapas de projeto, estando estes profissionais familiarizados com a visualização dos seus projetos em três dimensões. O uso da tecnologia BIM, porém, pode exigir novos processos na medida em que existe a necessidade de interoperabilidade entre as ferramentas de concepção e as de modelagem BIM que darão continuidade nas etapas posteriores do projeto. Todo o processo de projeto poderá sofrer alterações com a aplicação desta nova tecnologia, demandando antecipação de informações, ou sobreposição de etapas.

Delatorre e Santos (2014) apresentam em um de seus estudos de caso, que os processos de trabalho estão sendo revistos. As fases de projeto no contexto BIM se sobrepõem e é necessário que se trabalhe mais nas etapas iniciais do projeto, enquanto fases intermediárias exigem menos trabalho ou, podem até ser eliminadas.

De acordo com Abaurre (2013), a tecnologia BIM foi introduzida com o intuito de implementar um processo de projeto colaborativo e integrado. A antecipação de atividades e decisões nos processos colaborativos do BIM leva à simultaneidade dos projetos com trabalhos de Engenharia de Planejamento e de Controle de Qualidade. Essa característica aumenta a quantidade de informações geradas nas etapas iniciais. 
Em REIS (2011) são apresentados os casos de quatro empresas de Arquitetura que adotaram a tecnologia BIM. Foi relatado que uma das mudanças importantes constatadas foi a alteração no prazo das etapas do projeto.

No processo convencional, a carga de trabalho do arquiteto seria menor nos estudos preliminares e aumentaria conforme o projeto se aproxima do executivo. No BIM, algumas informações técnicas precisam ser definidas em etapas iniciais do projeto, levando a decisões antecipadas e, por consequência, a uma carga maior de trabalho nestas etapas.

Podemos considerar a partir destes dados, a realidade da afirmação de Wong (2010). O autor afirma que uma implementação de sucesso requer esforços em direção a tecnologia, pessoas e processos e que estes esforços devem ser equivalentes. Com a implementação de qualquer ferramenta BIM, observamos mudanças na relação entre as equipes que passam a trabalhar de forma mais colaborativa.

Processos e protocolos padronizados serão necessários para a definição de responsabilidades e a condução de revisões e validações do projeto. Assim como, boas práticas serão necessárias para que o gerenciamento dos dados seja adequado à estrutura da equipe e aos requisitos do projeto (GU, 2010).

\subsection{MAPEAMENTO DOS PROCESSOS DE PROJETO versUS USOS DO BIM}

Um guia desenvolvido por pesquisadores da Pennsylvania State University (CIC, 2015) recomenda que para implementar o BIM com sucesso é necessário concentrar esforços no desenvolvimento de um documento chamado Plano de Execução do Projeto BIM.

Um Plano de Execução do Projeto BIM bem documentado garante que todas as partes estarão claramente cientes das oportunidades e responsabilidades associadas com a incorporação do BIM no fluxo de trabalho. Este plano deve definir os usos apropriados do BIM juntamente com os detalhes e a documentação dos processos.

Em Barison e Santos (2014), consta uma extensão da relação de usos do BIM apresentados em CIC (2015) que abrange os usos da tecnologia BIM não apenas por empresas de Arquitetura, mas também, por empresas construtoras e incorporadoras.

Dentre usos do BIM descritos em ambas estas referências, foram selecionados aqueles que têm relação direta com a elaboração de projetos executivos de Arquitetura, a saber: (a) Modelagem 3D; (b) Levantamento de Materiais e (c) Documentação do Projeto.

Outros usos do BIM como a coordenação de modelos 3D, planejamento de execução da obra e estimativas de custo, não foram abordados por não representarem uma realidade no dia-a-dia das empresas de projeto de Arquitetura estudas nesta pesquisa.

\section{METODOLOGIA DA PESQUISA}

A pesquisa utilizou como metodologia a coleta e análise de dados junto a dois escritórios de projeto de Arquitetura que utilizam as ferramentas BIM Graphsoft ArchiCAD e Autodesk Revit. A caracterização destes escritórios é descrita no próximo tópico deste artigo.

Também contribuíram para a elaboração desta pesquisa, consultores BIM, revendas de software e dados públicos disponíveis nos websites dos desenvolvedores destas ferramentas BIM.

As informações a respeito do uso das ferramentas BIM foram obtidas em cada empresa a partir de visitas realizadas durante um período de 6 meses. Estas visitas tiveram como objetivos o acompanhamento do desenvolvimento de projetos e a realização de entrevistas com seus diretores, gerentes de projeto e projetistas. 
Para as entrevistas foi elaborado um questionário de questões objetivas e subjetivas categorizadas em grupos que abordam tanto aspectos técnicos quanto estratégicos da utilização das ferramentas BIM nos negócios das empresas de projeto estudadas. O foco na elaboração dessas questões foi identificar o quanto as ferramentas BIM avaliadas são utilizadas de fato. O Quadro 1 apresenta o questionário que cada empresa entrevistada respondeu. Uma análise crítica das respostas a este questionário é feita no tópico 4.2 deste artigo.

\section{Quadro 1 - Questionário aplicado nas entrevistas}

\section{Categoria $\quad$ Utilização das ferramentas BIM nas fases do projeto}

1. A ferramenta BIM é utilizada na etapa de concepção de produto? Caso positivo, em qual nível (1 = não é usada, 2 = raramente usada, 3 = pouco usada, $4=$ muito usada, $5=$ sempre usada)? Caso negativo, explique o porquê da sua não utilização.

2. Ainda sobre a fase de concepção de produto, são utilizadas outras ferramentas CAD em conjunto com a ferramenta BIM? Caso positivo, como é realizada a comunicação entre a ferramenta BIM e estas ferramentas CAD? Cite eventuais dificuldades de comunicação entre a ferramenta BIM e estas ferramentas CAD.

3. Sobre o uso da ferramenta BIM em cada etapa do projeto. Pontue o nível de utilização usando 1 = não é usada, 2 = raramente usada, 3 = pouco usada, 4 = muito usada, 5 = sempre usada.

\section{Categoria $\quad$ Elaboração e usos do modelo BIM}

4. Quais recursos na ferramenta BIM são utilizados quando existe a necessidade montar várias frentes de trabalho no mesmo projeto?

5. A ferramenta BIM é utilizada para checagem de critérios de projeto, como por exemplo, no projeto legal (quadros de áreas, recuos, índices, etc.) ou no projeto executivo (especificação de materiais, regras de dimensionamento de escadas e rampas, etc.). Caso positivo, indique que critérios de projeto conseguem ser monitorados e que recursos na ferramenta BIM são utilizados para este fim.

6. No processo de extração de quantitativos a partir de modelo BIM, muitas vezes é necessário aplicar algumas regras de medição de material. Um exemplo é a regra de desconto de vãos de esquadria ao contabilizar áreas de paredes. Ao extrair as quantidades do modelo BIM estas regras de medição são consideradas? Caso positivo, como isto é resolvido na ferramenta? Caso negativo, explique o porquê deste detalhe não ser considerado no processo.

7. Sobre o uso da ferramenta BIM na etapa de detalhamento de projeto. Cite as eventuais dificuldades enfrentadas atualmente.

\section{Categoria $\quad$ Integração com outros projetistas/cliente}

8. Como é a interação entre os projetistas de Estrutura e Arquitetura em relação ao uso da tecnologia BIM no desenvolvimento de um projeto? Cite as eventuais dificuldades enfrentadas atualmente.

9. Como ocorre o processo de revisão de modelos BIM? Cite eventuais dificuldades enfrentadas atualmente.

10. Em que formato de arquivo o cliente normalmente requisita a entrega dos projetos? Cite eventuais dificuldades enfrentadas atualmente.

Fonte: Os autores, 2015

Os critérios de avaliação das ferramentas BIM adotados foram baseados na experiência profissional dos autores na implementação e utilização da tecnologia BIM em seus respectivos negócios.

Os dados obtidos foram utilizados para a elaboração de uma planilha contendo o mapeamento dos usos do BIM abordados nesta pesquisa e as funcionalidades existentes nas ferramentas BIM estudadas. Como este mapeamento foi possível realizar uma avaliação técnica destas ferramentas quanto ao nível de conformidade aos processos BIM. 


\section{RESULTADOS DA PESQUISA}

\subsection{CARACTERIZAÇÃO DAS EMPRESAS DE PROJETO DE ARQUITETURA}

\subsubsection{Empresa A}

É subsidiária de uma empresa americana fundada em 1875. Localizada em São Paulo desde 1998 sua principal área de atuação concentra-se em projetos hospitalares e industriais em todas as fases de projeto: concepção, estudo preliminar, projeto legal e executivo. Seus projetos têm foco em inovação tecnológica e sustentabilidade.

Seu quadro de funcionários é composto por 1 diretor geral, 1 diretora de projetos, equipe de 10 arquitetos e uma equipe administrativa com 4 pessoas. Os dois diretores são arquitetos e responsáveis pela concepção dos projetos que são elaborados em conjunto com a empresa matriz.

A ferramenta BIM escolhida foi o Autodesk Revit em função da empresa matriz já utilizá-la. A tecnologia foi implementada na empresa com o objetivo de aumento de produtividade, mais confiabilidade nas informações no projeto e, principalmente, como estratégia competitiva. Um Consultor BIM auxiliou no processo de implementação que foi conduzido no período de 1 ano, com a criação de bibliotecas e templates personalizados ao processo de trabalho da empresa. A consultoria BIM juntamente com alguns membros da equipe de projeto da empresa foram os responsáveis pela elaboração de 2 projetos pilotos. No momento todos os projetos da empresa são elaborados utilizando a tecnologia BIM.

\subsubsection{Empresa B}

A empresa foi fundada em meados de 1980. Localizada em São Paulo sua principal área de atuação concentra-se em residências de alto padrão em todas as fases de projeto: concepção, estudo preliminar, projeto legal e executivo. Seus projetos têm foco em funcionalidade, conforto e estética.

Seu quadro de funcionários é composto por um arquiteto titular e uma equipe de quinze arquitetos, sendo que um deles acumula o cargo de Gerente BIM (BIM Manager).

A ferramenta BIM escolhida para desenvolver projetos executivos de Arquitetura foi o Graphisoft ArchiCAD. A empresa passou por dois momentos de implementação. Segundo o dono da empresa, no primeiro momento ocorrido em 2004, a falta de padronização na metodologia de trabalho gerou uma queda brusca de produtividade, pois cada arquiteto adotava seu próprio método de trabalho. A equipe de projeto precisou ser redimensionada para cumprir prazos e finalizar os projetos. A empresa sofreu financeiramente com gastos mais elevados nesta fase, enquanto não obtinha o lucro dos investimentos realizados em implementação BIM.

Em um segundo momento de implementação BIM, em 2009, o coordenador da equipe ficou responsável por retomar a experiência com uma reformulação geral da equipe e foi realizada uma análise detalhada dos processos. Foi desenvolvido um template único de trabalho, com padronização de elementos gráficos e uma listagem de informações que deveriam constar em cada etapa de trabalho.

Foram diagnosticados os problemas da empresa em seis meses e novos processos foram implantados nos seis meses seguintes. A partir daí, o que se viu foi um ganho de produtividade, enxugamento da equipe e aumento da qualidade dos trabalhos. 


\section{1}

\subsection{RESULTADOS DAS ENTREVISTAS COM AS EMPRESAS DE PROJETO DE ARQUITETURA}

Nos Quadros 2, 3 e 4 são apresentados resumos do que cada empresa respondeu para cada categoria de questão da entrevista. A empresa A utiliza o Autodesk Revit e a empresa B utiliza o Graphisoft ArchiCAD.

Quadro 2 - Utilização das ferramentas BIM nas fases do projeto

\begin{tabular}{|l|l|}
\hline \multicolumn{1}{|c|}{ Empresa A } & \multicolumn{1}{c|}{ Empresa B } \\
\hline A ferramenta BIM não é utilizada na fase de & A concepção do produto é realizada usando \\
concepção de produto, pois a matriz da empresa & técnicas tradicionais (croquis feitos a mão). \\
não utiliza o Revit nesta fase. & A ferramenta BIM é utilizada já nas fases iniciais \\
para validar o conceito do produto. \\
A concepção do produto é realizada usando \\
A ferramenta CAD-3D Sketchup é utilizada para \\
elaborar modelo 3D inicial que é usado para & $\begin{array}{l}\text { Foi relatado que existe um template de projeto } \\
\text { muito bem formatado elaborado pelo escritório }\end{array}$ \\
validar o conceito do empreendimento. & Em todas as demais fases (Ante-projeto, Projeto \\
A partir deste modelo 3D são extraídas plantas & Legal, Projeto Executivo) o ArchiCAD também é \\
para a ferramenta AutoCAD. & utilizado. \\
Após a validação destas plantas o modelo BIM é & Não são utilizadas outras ferramentas CAD \\
iniciado no Revit utilizando estas plantas como & 2D/3D apenas a ferramenta BIM. \\
referência. & \\
Os usuários relataram conhecer os recursos & \\
disponíveis no Revit para auxiliar nas fases \\
iniciais do projeto, mas não usam porque \\
precisam seguir o padrão de trabalho exigido \\
pela matriz.
\end{tabular}

Fonte: Os autores, 2015

\section{Quadro 3 - Elaboração e usos do modelo BIM}

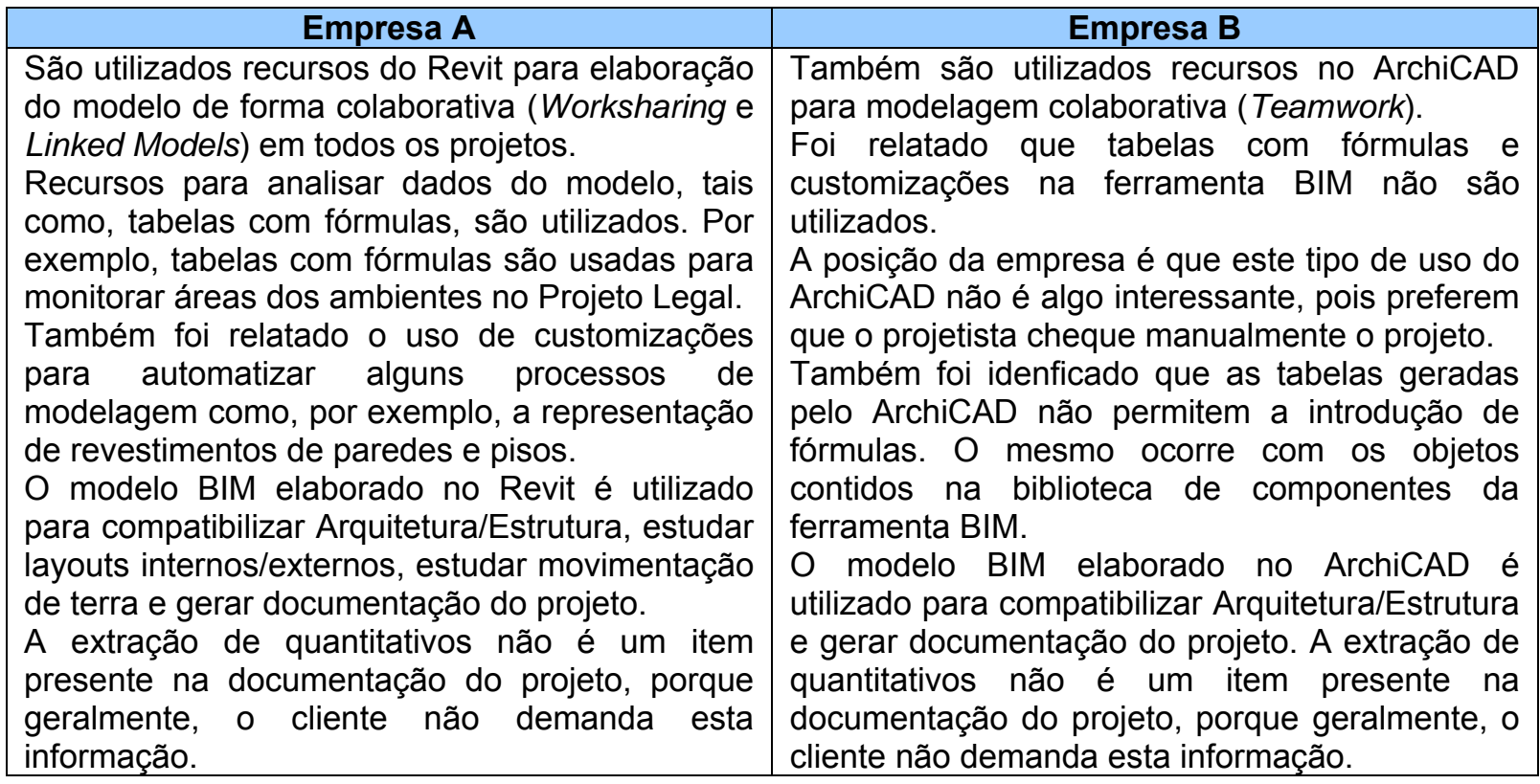


Quadro 4 - Integração com outros projetistas/cliente

\begin{tabular}{|l|l|}
\hline \multicolumn{1}{|c|}{ Empresa A } & \multicolumn{1}{|c|}{ Empresa B } \\
\hline A interação com os projetistas de Estrutura em & A troca de arquivos com o projetista de Estrutura \\
geral ocorre por meio de plantas no formato & é feita usados os formatos DWG e/ou DXF. \\
DWG que são vinculadas ao modelo 3D & Também é comum receber dos projetistas de \\
elaborado no Revit. & Estrutura modelos 3D no formato IFC. \\
As vezes arquivos IFC, originados da ferramenta & O conteúdo dos arquivos IFC é aproveitado no \\
TQS, são recebidos dos projetistas de Estrutura & ArchiCAD sem problemas. \\
e dificuldades para utilizar estes dados destes & O processo de revisão dos modelos BIM é feito \\
arquivos no Revit surgem. Verificou-se que estas & utilizando as ferramentas de revisão padrão do \\
dificuldades estão mais relacionadas com a falta & ArchiCAD. \\
de conhecimento dos usuários sobre o que é o & Nesse processo são realizadas reuniões com \\
IFC. & cliente para revisão do projeto usando o modelo \\
No caminho inverso (empresa A/projetista de & BIM. \\
Estrutura) são trocados arquivos no formato & As solicitações do cliente são atendidas em uma \\
DWG gerados a partir do modelo 3D elaborado & nova revisão deste modelo. Eventualmente, é \\
no Revit. & utilizado o recurso BIMx para gerar um arquivo \\
As revisões nos modelos BIM são feitas a partir & de visualização para cliente navegar pelo \\
da etapa de Anteprojeto. Não relataram & projeto. \\
dificuldades técnicas para essas revisões, pois & Os projetos são entregues ao cliente nos \\
os arquivos são enviados em formato 2D para o o & formatos PDF e DWG, também é entregue um \\
cliente e eventualmente algumas imagens 3D & arquivo que permite a navegação interativa pelo \\
são enviadas para a melhor compreensão das & projeto (recurso BIMx). \\
soluções adotadas. Os clientes não fazem uso & \\
do modelo 3D. & \\
Os projetos são entregues aos clientes nos & \\
formatos PDF e DWG. Não existem dificuldades & \\
atualmente, mas, existe o desejo de que os & \\
clientes sejam capazes de manipular os modelos & \\
3D para compreenderem melhor as soluções de & \\
projeto escolhidas e obter maior participação & \\
durante o processo. & \\
\hline &
\end{tabular}

Fonte: Os autores, 2015

\subsection{MAPEAMENTO DOS USOS DO BIM versus FUNCIONALIDADES NAS FERRAMENTAS BIM}

O Autodesk Revit 2015 e o Graphisoft ArchiCAD 18 foram as ferramentas BIM avaliadas nesse estudo. Foram escolhidas versões contemporâneas destas duas ferramentas para uma avaliação mais justa.

Ao avaliar tecnicamente estas ferramentas procurou-se identificar quais funcionalidades elas disponibilizavam para atender os usos do BIM abordados nesta pesquisa. Desta forma, obteve-se um mapeamento das funcionalidades com os usos do BIM e uma visão quantitativa dos recursos disponíveis nas ferramentas avaliadas.

Para realizar este mapeamento de Usos do BIM versus Funcionalidades das Ferramentas BIM, baseado na experiência dos autores na utilização e implementação desta tecnologia, foram selecionadas as principais atividades envolvidas na utilização destas ferramentas neste contexto.

Foram levantadas em cada ferramenta, as funcionalidades relacionadas com as atividades selecionadas. O Quadro 5 apresenta uma relação das atividades consideradas para cada uso do BIM abordado neste trabalho, bem como a quantidade de funcionalidades levantadas em cada ferramenta BIM para atender estas atividades. 
Quadro 5 - Mapeamento Usos do BIM vs Funcionalidades das Ferramentas BIM

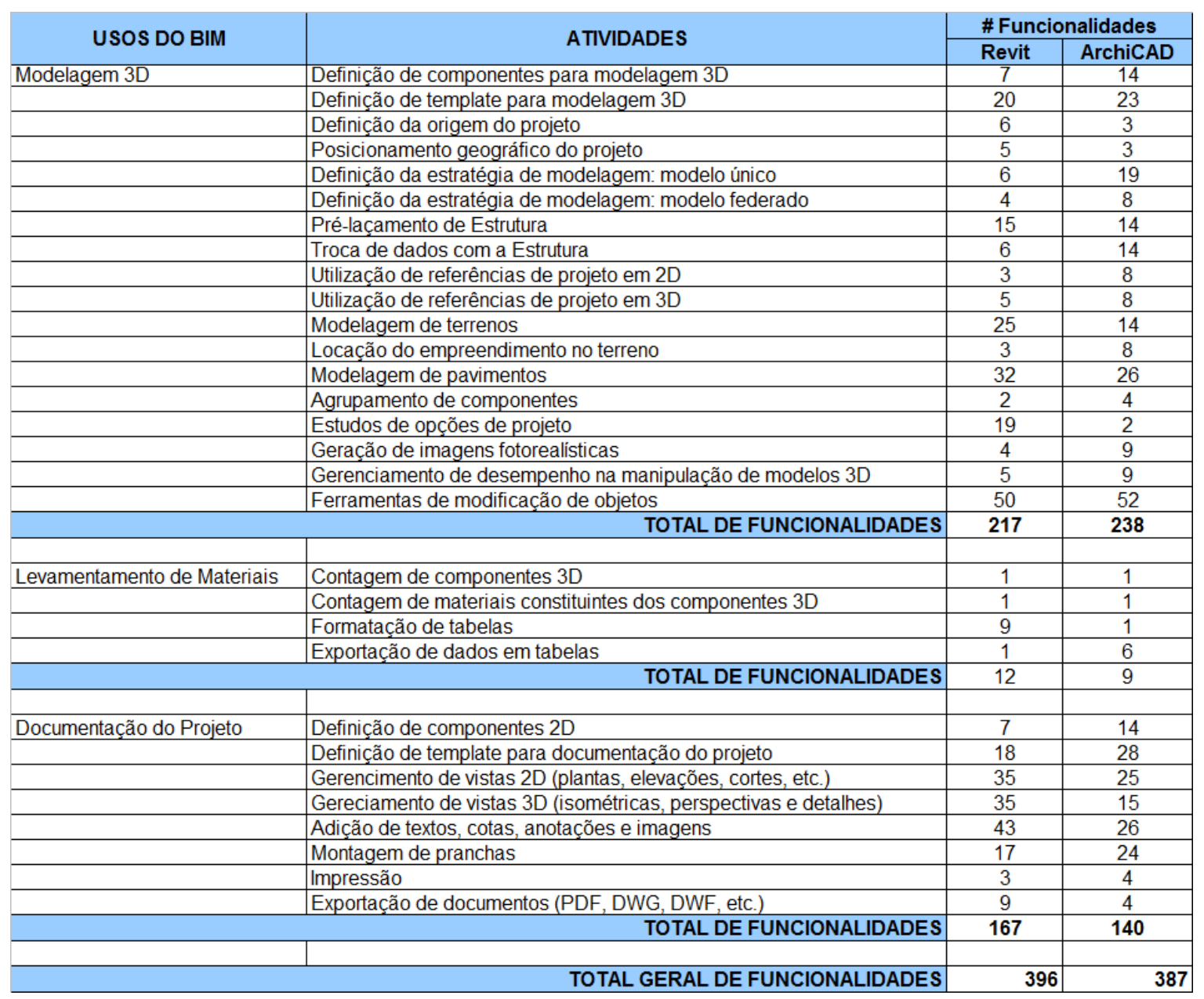

Fonte: Os autores, 2015

Este levantamento mostrou que é mínima a diferença entre o número de funcionalidades oferecidas por ambas as ferramentas BIM para atender as necessidades dos usos do BIM abordados nessa pesquisa. As quantidades ficaram relativamente equilibradas, demonstrando que ambas as ferramentas BIM oferecem recursos para atender todas as atividades consideradas neste estudo.

Além de dados técnicos foram levantados dados comerciais das ferramentas BIM avaliadas nesta pesquisa. Os dados apresentados no Quadro 6 foram obtidos em duas revendas autorizadas de cada software.

Quadro 6 - Valores de investimento para aquisição de licenças

\begin{tabular}{|l|lr|lr|rr|}
\multirow{2}{*}{ Ferramentas BIM } & \multicolumn{4}{c|}{ Modelos de Licenciamento (valores em dólar) } \\
\cline { 2 - 7 } & \multicolumn{2}{|c|}{ Permanente } & \multicolumn{1}{|l|}{ Assinatura Anual } & \multicolumn{2}{c|}{ Aluguel } \\
\hline Autodesk Revit & USD & $8.638,00$ & USD & $1.373,00$ & USD & $3.668,00$ \\
\hline Graphisoft ArchiCAD & USD & $3.589,74$ & USD & 769,23 & USD & $1.794,87$ \\
\hline
\end{tabular}

Fonte: Os autores, 2015

Em relação aos dados comerciais das ferramentas BIM avaliadas neste estudo, observou-se que o ArchiCAD apresenta maior flexibilidade de negociação para aquisição de licenças que o Revit, o qual demanda um investimento substancialmente maior. 
A diferença nos valores das licenças entre as ferramentas BIM poderia ser justificada pelo fato do Autodesk Revit possuir um número de funcionalidades que permitem aos seus usuários a elaboração não só de modelos BIM de Arquitetura, mas também, de outras disciplinas como Estrutura e Instalações Prediais (AUTODESK, 2015).

Por outro lado, O ArchiCAD é uma ferramenta BIM mais especializada na elaboração de modelos BIM de Arquitetura. Entretanto, nas últimas versões do ArchiCAD, a Graphsoft já iniciou a introdução de recursos para modelagem de Instalações Prediais (GRAPHSOFT, 2015). Uma indicação de que este desenvolvedor pretende ampliar as funcionalidades desta ferramenta para atender usuários de outras disciplinas de projeto.

\section{CONCLUSÕES}

A análise de todo material levantado nessa pesquisa apresentou vários aspectos interessantes. Primeiramente, a limitação por parte dos usuários na utilização de todos os recursos disponíveis nas duas ferramentas BIM avaliadas.

O uso superficial das ferramentas BIM dentro das empresas estudadas, decorrente, ou a falta de interesse dos usuários em se aprofundar nos recursos disponíveis ou, ao treinamento deficiente, levou a subtilização destas ferramentas.

Observou-se certa resistência dentro das empresas de projeto entrevistadas em abandonar definitivamente processos tradicionais como, por exemplo, a elaboração de croquis a mão para a concepção de novos projetos.

Apesar de alguns entrevistados relatarem conhecer recursos nas ferramentas BIM para concepção de produto, notou-se que outras ferramentas CAD 3D são utilizadas para este fim. O que não poderia ser considerado um procedimento incorreto desde que, os dados gerados nestas outras ferramentas fossem reaproveitados de forma mais adequada. Neste cenário, por exemplo, notou-se que ao invés de reaproveitar o dado gerado na ferramenta CAD 3D genérica (Sketchup), o mesmo foi recriado na ferramenta BIM (Revit).

Os procedimentos adotados na empresa A não demonstram que a ferramenta BIM seja deficiente, mas que o processo poderia ser revisto de forma a garantir melhor consistência com a tecnologia BIM.

Outro dado que chamou atenção foi a quantidade de funcionalidades oferecidas por ambas as ferramentas para atender as necessidades dos usos do BIM abordados nessa pesquisa. As quantidades ficaram relativamente equilibradas, demonstrando que ambas as ferramentas BIM oferecem recursos para atender todas as atividades consideradas neste estudo.

Em relação aos dados comerciais das ferramentas BIM avaliadas neste estudo, observou-se que o ArchiCAD apresenta maior flexibilidade de negociação para aquisição de licenças que o Revit, o qual demanda um investimento substancialmente maior.

Sob o ponto de vista dos usuários das ferramentas BIM, alguns dos entrevistados que conhecem as duas ferramentas, relataram que o ArchiCAD oferece maior liberdade na tarefa de modelagem 3D que o Revit.

Também foi identificado no ArchiCAD, que a criação de novas bibliotecas com objetos paramétricos avançados que incluem fórmulas que possibilitem cálculos matemáticos, com resultados que influenciam não só a geometria, mas também na aparência dos componentes, requer conhecimentos de programação dos usuários.

No Revit a criação de componentes com estas características não requer estes conhecimentos dos seus usuários, o que permite incluir maior inteligência nos modelos BIM. Mas, notou-se que os usuários entrevistados ainda não chegaram neste nível de utilização 
de suas ferramentas BIM, ou por não conhecerem estas possibilidades, ou por não visualizarem a necessidade de adicionar mais inteligência em seus modelos BIM.

Concluí-se, portanto, que as duas ferramentas BIM avaliadas atendem aos processos BIM associados ao projeto executivo de Arquitetura que foram abordados nesta pesquisa: Modelagem 3D, Levantamento de Materiais e Documentação do Projeto. A preferência de escolha dos usuários entre estas ferramentas BIM se dá mais pela facilidade em sua aquisição, aprendizado e utilização.

\section{REFERÊNCIAS}

ABAURRE, M. W. Modelos de Contrato Colaborativo e Projeto Integrado para Modelagem da Informação da Construção. 2014. 225 p. São Paulo, 2013. Dissertação (Mestrado). Escola Politécnica, Universidade de São Paulo, São Paulo, 2014.

ARAYICI Y., COATES P., KOSKELA, L., KAGIOGLOU, M. BIM adoption and implementation for architectural practices, Structural Survey. Emerald Group Plubishing, Bingley, UK, Vol. 29 No. 1, 2011, p. 7-25.

ASBEA. Manual de Escopo de Projetos e Serviços de Arquitetura e Urbanismo. 2012, Versão 2. Disponível em: <http://migre.me/rcTD8>. Acesso em: 15 jul. 2015

AUTODESK. Building Design Software | Revit Family | Autodesk. Disponível em: <http://www.autodesk.com/products/revit-family/overview/>. Acesso em: 15 jun. 2015.

BARISON, M. B., SANTOS, E. T. Ferramenta para planejamento de disciplina BIM. In: XV Encontro Nacional da Tecnologia do Ambiente Construído, 2014, Maceió. Anais... Maceió: UFAL, 2014. p. 2933-2942.

CIC - Computer Integrated Construction Research Program. BIM Project Execution Planning Guide. The Pennsylvania State University, University Park, PA, USA. Version 2.1, 2011. Disponível em: <http://bim.psu.edu/default.aspx>. Acesso em: 15 jun. 2015.

DELATORRE, J. P. M., SANTOS, E. T. Introdução de novas tecnologias: o caso do BIM em empresas de construção civil. In: XV Encontro Nacional da Tecnologia do Ambiente Construído, 2014, Maceió. Anais... Maceió: UFAL, 2014. p. 2842-2851.

GRAPHSOFT. About ARCHICAD - A 3D architectural BIM software for design \& modeling. Disponível em: <http://www.graphisoft.com/archicad/>. Acesso em: 15 jun. 2015.

GU, N. LONDON, K. Understanding and facilitating BIM adoption in the AEC industry. Automation in Construction Journal, Vol. 19, No. 8, 2010, p. 988-999.

JERNIGAN, F. BIG Bim, Little Bim: The practical approach to building information modeling. Integrated Practice Done the Right Way! $1^{\text {a }}$ ed., Salisbury: 4Site Press, 2007. 323 p.

NAKAMURA, J. Como anda o BIM nas incorporadoras. Revista Construção Mercado Negócios de Incorporação e Construção. Ed. 143, jun. 2013. Disponível em: <http://migre.me/rcTGr>. Acesso em: 15 jun. 2015.

REIS, P. Saiba como foi a implementação do BIM em escritórios de arquitetura. Revista Arquitetura e Urbanismo. Ed. 208, jul. 2011. Disponível em: <http://migre.me/rcZFN>. Acesso em: 15 jun. 2015

SOUZA, F; WYSE, M e MELHADO, S. B. The Brazilian Design Manager Role and Responsabilities after the BIM Process Introduction. In: CIB World Building Congress 2013, Brisbane, 2013. Proceedings... Brisbane: Queensland University of Technology, 2013. v. 19.

WONG, A; WONG, F; NADEEM, A. Attributes of Building Information Modelling Implementations in Various Countries. Architectural Engineering and Design Management Journal,Vol. 6, No. 4, 2010, p. 288-302. 